LETTER TO JMG

\section{A large patient study confirming that facioscapulohumeral muscular dystrophy (FSHD) disease expression is almost exclusively associated with an FSHD locus located on a $4 q A$-defined

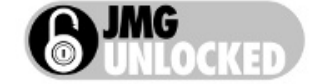
4qter subtelomere

\author{
N S T Thomas, K Wiseman, G Spurlock, M MacDonald, D Üstek, \\ $M$ Upadhyaya
}

This article is available free on JMG online via the JMG Unlocked open access trial, funded by the Joint Information Systems Committee. For further information, see http://jmg.bmj.com/cgi/content/full/42/ $2 / 97$
Facioscapulohumeral muscular dystrophy (FSHD), an autosomal dominant disorder, represents the third most common human muscular dystrophy. The FSHD disease locus, at chromosome $4 \mathrm{q} 35$, is associated with large contractions of the polymorphic repeat sequence array D4Z4. In addition to FSHD disease association with large D4Z4 deletions, a biased interaction with a specific 4 gter subtelomeric sequence has been described in patients. Two distinct 4 qter subtelomeres, defined as types $4 \mathrm{qA}$ and $4 \mathrm{qB}$, have been identified and shown to be equally prevalent in the Caucasian population. In almost all 4q35-linked patients with FSHD, however, disease expression only occurs when large D4Z4 deletions are located on $4 q A$-defined $4 q$ ter subtelomeres. Conversely, large D4Z4 repeat contractions situated on $4 \mathrm{qB}$-defined subtelomeres either are not disease-causing or exhibit a greatly reduced disease penetrance. This study was initiated to confirm this direct FSHD disease association data by measuring the frequency of type $4 \mathrm{qA}$-defined and $4 \mathrm{qB}$-defined subtelomeric sequences in a large cohort of 164 unrelated patients with FSHD from Turkey and the UK, all known to have large D4Z4 deletions. An almost complete association was found between large D4Z4 repeat array deletions located on $4 \mathrm{qA}$-defined $4 \mathrm{qter}$ subtelomeres and disease expression in our large FSHD patient cohort. The observed failure of probes $4 q A$ and $4 q B$ to hybridise to two patient-derived DNA samples confirms the presence of an additional rare type of 4 qter subtelomeric sequence in humans.

F acioscapulohumeral muscular dystrophy (FSHD), an autosomal dominant muscle disorder in which patients display a progressive weakness of the musculature of the face, shoulders and upper arms, ${ }^{1}$ is the third most common human muscular dystrophy. FSHD has an estimated incidence of at least 1:20 000 livebirths and exhibits a high level of new mutations, which represent $10-30 \%$ of all new FSHD cases. ${ }^{2}$ A high degree of intra-familial and inter-familial clinical variability is exhibited by patients, with affected individuals often expressing a range of clinical phenotypes, from severe neonatalonset forms, through "classical" FSHD, to asymptomatic or non-penetrant gene carriers, sometimes all within the same family. ${ }^{3}$

The FSHD locus is located at $4 \mathrm{q} 35$ adjacent to the 4qter subtelomeric region. ${ }^{4}$ A variable number tandem repeat polymorphic locus, designated D4Z4, was identified in this region, which in normal individuals usually contains multiple integral copies $(11>100)$ of a $3.3 \mathrm{~kb} K p n \mathrm{I}$ tandem repeat sequence. ${ }^{5}$ However, in the majority of patients with FSHD, large integral deletions of individual $K p n I$ repeats reduce the size of this $D 4 Z 4$ repeat arrays down to less than 11 repeats $(\leqslant 38 \mathrm{~kb})$. No specific FSHD gene has yet been identified, nor has the underlying pathological mechanism(s) involved in disease expression been explained. An approximate inverse correlation has been reported ${ }^{6} 7$ between the exact number of D4Z4 repeats retained by a patient and the level of disease severity exhibited by that patient, although the clinical variability associated with FSHD may often confound this relationship.

Two distinct 4qter subtelomeres, designated $4 \mathrm{qA}$ and $4 \mathrm{qB}$, have been recognised ${ }^{8}$ and are identified by hybridisation of specific $4 \mathrm{qA}$ and $4 \mathrm{qB}$ probes to genomic sequences located immediately distal of the D4Z4 repeat array. The 4qA-defined 4qter subtelomere contains an $\sim 8 \mathrm{~kb} \beta$-satellite sequence (fig l) that shows significant sequence homology to the 10qter subtelomere, and hybridisation with the 4qA probe identifies genomic sequences from both subtelomeric regions. The 4qAdefined and $4 \mathrm{qB}$-defined $4 \mathrm{qter}$ subtelomeres are found to occur with almost equal frequency in the Caucasian population. ${ }^{8}$ However, essentially all patients with FSHD exhibit highly biased $4 \mathrm{qA} / 4 \mathrm{qB}$ allelic frequencies, in which disease expression is not only associated with large D4Z4 contractions, but that these small $(\leqslant 38 \mathrm{~kb}) \quad D 4 Z 4$-containing EcoRI/BlnI fragments must be specifically located on a 4qA-defined 4qter subtelomere. ${ }^{8}$ Conversely, small EcoRI/BlnI fragments that are located either on a 4qB-defined 4qter subtelomere or on a 4qA-defined loqter subtelomere are either not disease-associated, or they show a greatly reduced disease penetrance. ${ }^{10}$ In a very small number of DNA samples analysed, both from patients with FSHD and from normal individuals, the small 4q35-derived D4Z4-containing HindIII fragments failed to hybridise to either probe $4 \mathrm{qA}$ or $4 \mathrm{qB}$, indicating the possible presence of an additional 4qter subtelomeric type in humans. ${ }^{11}$

This large patient study was therefore undertaken to confirm that it is only the large D4Z4 deletions located on a 4qA-defined 4qter subtelomeres that are associated with FSHD disease expression. The study involved the analysis of DNA samples from 164 unrelated affected individuals, along with a smaller number of normal individuals. Each of the patients with FSHD involved in the study was known to carry a small, diseaseassociated, 4q35-derived EcoRI/BlnI fragment, as identified by

Abbreviations: FSHD, facioscapulohumeral muscular dystrophy; LGE, linear gel electrophoresis; PFGE, pulsed-field gel electrophoresis 


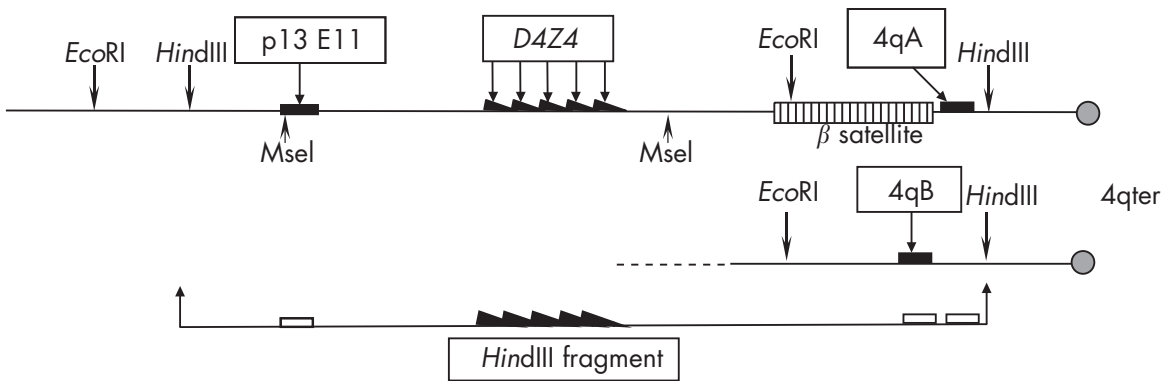

Figure 1 Genomic map of the facioscapulohumeral muscular dystrophy locus region containing $4 \mathrm{qA}$-defined and 4qB-defined 4qter subtelomeres. A schematic map of the $4 q$ ter subtelomeric region. The location of the highly variable D4Z4 repeat array, the position of the p13-E1 1, p4qA and p4qB-defined sequences, the location of the relevant restriction enzyme sites and the position of $\sim 8 \mathrm{~kb} \beta$-satellite sequence that defines the $4 \mathrm{qA}$-containing $4 \mathrm{qter}$ subtelomere are shown. The large D4Z4-containing Hindlll fragment identified by $p 4 q A$ and $p 4 q B$ hybridisation is also shown.

hybridisation to probe pl3-E11. Probes 4qA and 4qB were hybridised to HindIII-digested genomic DNA, from patients and controls, separated either by pulsed-field gel electrophoresis (PFGE) or by standard linear gel electrophoresis (LGE), to determine the $4 \mathrm{q} 35$-located $4 \mathrm{qA} / 4 \mathrm{qB}$ allele frequencies and to correlate this information with the presence of small, 4q35located $D 4 Z 4$ repeat arrays.

\section{PATIENTS AND METHODS FSHD patients}

The FSHD patient cohort contained DNA samples from 164 unrelated affected individuals, either obtained for FSHD research purposes or from those patients referred to our molecular diagnostic facility for FSHD analysis. Most patients were from the UK (144), with 15 patients from Turkey and five from Australia. The patients included in the study all conformed to accepted FSHD clinical criteria.

High molecular weight DNA (HMW-DNA) was available for PFGE from 15 Turkish and 50 British patients with FSHD, and from 15 Turkish and 35 British normal individuals. Standard DNA, suitable for linear gel electrophoretic analysis, was available from a further 99 unrelated patients with FSHD, of which 35 were familial disease cases. Control DNA from 50 normal individuals was also available.

All the FSHD patients had previously undergone DNA diagnosis and had been identified as carrying small $(\sim 38 \mathrm{~kb}$, $\leqslant 11$ repeats), $4 \mathrm{q} 35$-located $D 4 Z 4$ repeat arrays, as determined by pl3E-11 hybridisation to EcoRI-digested and EcoRI/BlnIdigested genomic DNA. ${ }^{12}$

\section{DNA isolation, analysis of D4Z4 repeat size and $4 q A$ and $\mathbf{4 q B}$ variant determination}

Standard and high molecular weight DNA was isolated from peripheral blood lymphocytes from patients with FSHD and controls using previously published methods. ${ }^{13}$ For PFGE analysis, $5 \mu \mathrm{g}$ HMW-DNA was used for each restriction digest. The 4qA-defined and 4qB-defined subtelomeric alleles were identified by the sequential hybridisation of the probes p4qA and $\mathrm{p} 4 \mathrm{qB}$ to HindIII-digested DNA separated on a $0.4-0.5 \%$ agarose gel electrophorised at $28 \mathrm{~V}$ for $72-48 \mathrm{~h}$ and blotted on to hybond membrane. ${ }^{14}$

\section{RESULTS}

All 164 unrelated patients with FSHD were previously identified as having small $(\leqslant 38 \mathrm{~kb}), 4 \mathrm{q} 35$-located EcoRI/BlnI fragments. No evidence for somatic mosaicism involving this small $E c o$ RI/B $\ln \mathrm{I}$ fragment was found in any of the unaffected parents of affected sporadic patients with FSHD who were fully investigated. In many of these de novo cases, only the patient's DNA was available for a complete screen with pl3-El1, and the
$4 \mathrm{qA}$ and $4 \mathrm{qB}$ probes. Xapl digests of the DNA samples were not routinely carried out, precluding determination of 10qA allele frequencies.

DNA samples from normal individuals, of both Turkish and UK origin, were all found to display equivalent frequencies for the $4 \mathrm{q} 35$-located $4 \mathrm{qA}$ and $4 \mathrm{qB}$ markers, with $53 \% 4 \mathrm{qA}$ and $47 \%$ $4 \mathrm{qB}$ alleles found overall. The study also showed that either HindIII-digested HMW-DNA analysed by PFGE or HindIIIdigested standard DNA analysed by LGE was equally proficient in resolving 4qA-specific and 4qB-specific bands. The increased gel-band resolution that is achievable by PFGE-based analysis permits much greater accuracy when sizing the different DNA restriction fragments on the gel, hence this system is the method of choice routinely used for FSHD DNA analysis in the molecular diagnostic laboratory.

Figure 2 shows an example of the hybridisation results for probe pl3-Ell to EcoRI and EcoRI/BlnI-digested DNA and probes p4qA and p4qB to HindIII-digested DNA from a typical patient with FSHD. PFGE-based analysis of 65 unrelated patients with FSHD, 15 from Turkey and 50 from the UK, showed the complete concurrence of a small $(\leqslant 11$ repeats) D4Z4 array located on 4qA-defined 4q35-located subtelomere in each patient. However, analysis of the normal chromosome 4 in each of these 65 patients showed an almost equal frequency for the $4 \mathrm{qA}(54.5 \%)$ and $4 \mathrm{qB}(45.5 \%)$ alleles. In the more extensive LGE-based analysis of HindIII-digested standard DNA from 99 unrelated patients with FSHD, it was found that 97 of these samples exhibited a small 4q35-located 4qA allele compatible with their small $(\sim 38 \mathrm{~kb}, \leqslant 11$ repeats $), E c o R I / B \ln I$ fragment. However, in the DNA from the two remaining patients with FSHD, neither probe 4qA nor probe 4qB hybridised to any small 4q35-specific DNA fragments, even though the expected number of non-specific gel bands were observed with both probes. Figure 3 shows the hybridisation results from applying probes pl3-E11, p4qA and p4qB to EcoRI/BlnI-digested and HindIII-digested DNA from one of these patients with FSHD .

\section{DISCUSSION}

Assessment of 4qA and 4qB allele frequencies in a large cohort of 164 unrelated patients with FSHD, which included 15 unrelated Turkish patients, confirmed the almost exclusive association between FSHD disease-expression and the presence of small ( $\sim 38 \mathrm{~kb}, \leqslant 11$ repeats) D4Z4 repeat arrays located on small 4qA-defined 4qter subtelomeres. The FSHD patient sample surveyed more than double the numbers reported in two previous similar studies. ${ }^{89}$ As none of the normal individuals analysed showed a small $(\leqslant 38 \mathrm{~kb}) 4 \mathrm{q} 35$-located $D 4 Z 4$ repeat array, we were unable to corroborate whether any of these were associated with a small 4qB-defined 4qter 


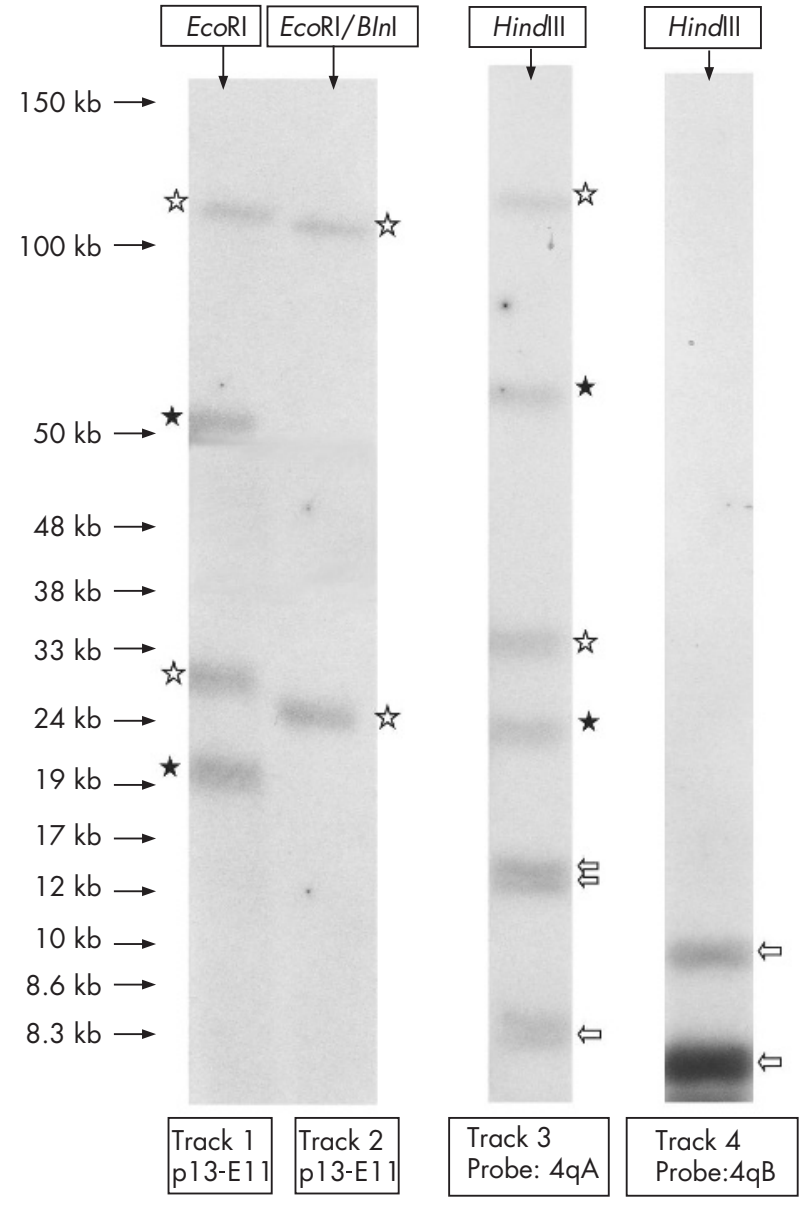

Figure 2 Pulsed-field gel electrophoresis analysis of patient with facioscapulohumeral muscular dystrophy (FSHD) DNA. EcoRI and EcoRI/ BInl DNA digests hybridised to p13-E1 1 (track 1 and 2). Hindlll DNA digests hybridised to probe $4 \mathrm{qA}$ (track 3 ) and probe $4 \mathrm{qB}$ (track 4). Chromosome 4-specific bands (white stars) and chromosome 10-specific bands (black stars) are indicated. All non-specific hybridising bands are identified (white arrows). The Hindlll-digested DNA hybridises only to probe $4 \mathrm{qA}$ (track 3 ) and not to probe $4 \mathrm{qB}$, indicating that this patient with FSHD has an AA genotype at $4 \mathrm{q} 35$.

subtelomere, and would therefore be considered to be nondisease-associated. ${ }^{10}$

The $4 \mathrm{q} 35$-located $4 \mathrm{qA} / 4 \mathrm{qB}$ allele frequencies determined in the 15 normal Turkish DNA samples showed an equal prevalency for $4 \mathrm{qA}$-defined and $4 \mathrm{qB}$-defined $4 \mathrm{qter}$ subtelomeres, showing no apparent difference in the $4 \mathrm{qA} / 4 \mathrm{qB}$ allele frequencies in this ethnic subpopulation.

An additional rare type of 4qter subtelomere was identified after the repeated failure of hybridisation of both probes 4qA and $4 \mathrm{qB}$ to any small $4 \mathrm{q} 35$-specific DNA fragment from two patients with FSHD, known to have small (24 and $21 \mathrm{~kb}$ ) $E c o R I / B \ln I$ fragments. None of the normal individuals analysed gave evidence for an alternative 4qter subtelomere, although this has been reported. ${ }^{11}$ It is not known if there is any relationship between this rare 4qter subtype and FSHD disease expression.

This large FSHD patient study has corroborated and strengthened the evidence for the almost exclusive association between disease expression and the location of the FSHD locus on a small 4qA-defined 4qter subtelomere. Confirmation of this essentially complete allelic association greatly increases confidence in implementing the use of the $4 \mathrm{qA}$ and $4 \mathrm{qB}$ probes in

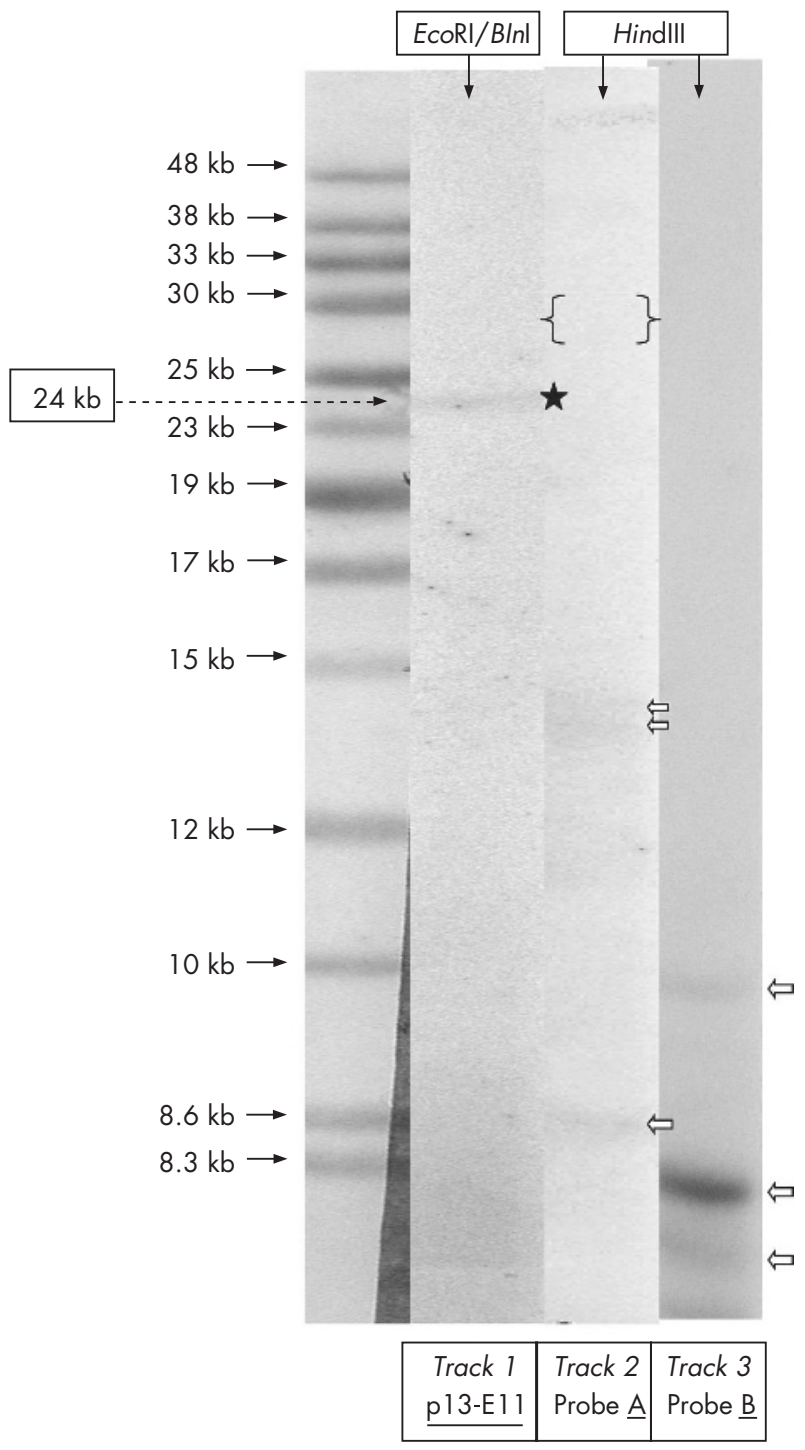

Figure 3 Analysis of DNA from a patient with facioscapulohumeral muscular dystrophy lacking $4 \mathrm{qA}$ and $4 \mathrm{qB}$ alleles. Track 1: DNA from patient digested with EcoRI/B/nl hybridised with p13-E1 1, a 4q35-derived $24 \mathrm{~kb}$ band is indicated (black star). Tracks 2/3: Patient DNA digested with Hindlll and hybridised to probe $4 \mathrm{qA}^{2}$ and probe $4 \mathrm{qB} .{ }^{3}$ No band comparable to the $24 \mathrm{~kb}$ EcoRl/BInl was found, an $\sim 30 \mathrm{~kb} 4 \mathrm{qA}$ band would be expected (within the region of the gel marked \{ \}). Only nonspecific hybridising bands are present (white arrows).

routine molecular diagnoses of FSHD. This may be especially important in those FSHD cases which show extreme variability in their clinical presentation.

\section{ACKNOWLEDGEMENTS}

We thank all the clinicians who have provided us with FSHD patient samples, especially Dr Peter Lunt (Bristol), Dr Mark Rogers (Cardiff), and Dr Piraye Serdaroglu (Turkey). We gratefully acknowledge the financial support of the Association Française contre les Myopathies (AFM). Finally, we thank the many patients with FSHD, and their families, who have for many years given us their full support and encouragement.

\section{Authors' affiliations}

N S T Thomas, K Wiseman, G Spurlock, M MacDonald, M Upadhyaya, Department of Medical Genetics, Cardiff University, School of Medicine, Heath Park, Cardiff, UK 
D Üstek, Division of Medical Genetics, Department of Pediatrics, Istanbul University, 34390, Capa, Istanbul, Turkey

Competing interests: None.

Correspondence to: Professor M Upadhyaya, Department of Medical Genetics, Cardiff University, School of Medicine, Heath Park, CF14 4XN, UK; upadhyaya@cardiff.ac.uk

Received 29 March 2006

Revised 31 July 2006

Accepted 22 August 2006

Published Online First 20 September 2006

\section{REFERENCES}

1 Padberg GW. Facioscapulohumeral disease [Thesis]. Leiden, The Netherlands: Leiden University, 1982.

2 Zatz M, Marie SK, Passos-Bueno MR, Vainzof M, Campiotto S, Cerqueira A, Wijmenga $C$, Padberg G, Frants R. High proportion of new mutations and possible anticipation in Brazilian facioscapulohumeral muscular dystrophy. Am J Hum Genet 1995:56:99-105.

3 Padberg, Facioscapulohumeral muscular dystrophy: a clinician's experience. In: Upadhyaya M, Cooper DN, eds. Facioscapulohumeral muscular dystrophy. Clinical medicine and molecular cell biology. Oxon: Garland Science/BIOS Scientific Publishers, 2004:41-54.

4 Wijmenga C, Padberg GW, Moerer P, Wiegant J, Liem L, Brouwer OF, Milner ECB, Weber JL, Ommen GJ van, Sandkuyl LA, Frants RR. Mapping of facioscapulohumeral muscular dystrophy gene to chromosome 4q35-qter by multipoint linkage analysis and in situ hybridization. Genomics 1991;9:570-5

5 van Deutekom JC, Wijmenga C, Tienhoven EA van, Gruter AM, Hewitt JE, Padberg GW, Ommen GJ van, Hofker MH, Frants RR. FSHD associated DNA rearrangements are due to deletions of integral copies of a $3.2 \mathrm{~kb}$ tandemly repeated unit. Hum Mol Genet 1993;2:2037-42.

6 Lunt PW, Jardine PE, Koch MC, Maynard J, Osborn M, Williams M, Harper PS Upadhyaya M. Correlation between fragment size at D4F104S1 and age at onset or at wheelchair use, with a possible generational effect, accounts for much phenotypic variation in 4q35- facioscapulohumeral muscular dystrophy (FSHD). Hum Mol Genet 1995;4:951-8.

7 Tawil R, Forrester J, Griggs RC, Mendell J, Kissel J, McDermott M, King W, Weiffenbach B, Figlewicz D. Evidence for anticipation and association of deletion size with severity in facioscapulohumeral muscular dystrophy. The FSHDYGroup. Ann Neurol 1996;39:744-8.

8 Lemmers RJ, Kievit P de, Sandkuil L, Padberg GW, Ommen GJ van, Frants RR, Maarel SM van der. Facioscapulohumeral muscular dystrophy is uniquely associated with one of the two variants of the $4 \mathrm{q}$ subtelomere. Nat Genet 2002;32:235-6.

9 van Geel M, Dickson MC, Beck AF, Bolland DJ, Frants RR, van der Maarel SM, de Jong PJ, Hewitt JE. Genomic analysis of human chromosome $10 q$ and $4 q$ telomeres suggests a common origin. Genomics 2002;79:210-17.

10 Lemmers RJ, Wohlgemuth M, Frants RR, Padberg GW, Morava E, van der Maarel SM. Contractions of D4Z4 on $4 \mathrm{qB}$ subtelomeres do not cause

facioscapulohumeral muscular dystrophy. Am J Hum Genet 2004;75:1124-30.

11 Buzhov BT, Lemmers RJ, Tournev I, Dikova C, Kremensky I, Petrova J, Frants RR, van der Maare SM. Recurrent somatic mosaicism for D4Z4 contractions in a family with facioscapulohumeral muscular dystrophy. Neuromuscul Disord 2005; 15:471-5

12 Upadhyaya M, Maynard J, Rogers MT, Lunt PW, Jardine P, Ravine D, Harper PS Improved molecular diagnosis of facioscapulohumeral muscular dystrophy (FSHD): validation of the differential double digestion for FSHD. J Med Genet 1997:34:476-9.

13 Upadhyaya M, MacDonald M, Ravine D. Prenatal diagnosis for facioscapulohumeral muscular dystrophy (FSHD). Prenat Diagn 1999;19:959-65.

14 Lemmers RJ, Osborn M, Haaf T, Rogers M, Frants RR, Padberg GW, Cooper DN van der Maarel SM, Upadhyaya M. D4F104S1 deletion in facioscapulohumeral muscular dystrophy: phenotype, size, and detection. Neurology 2003;61:178-83.

\section{bmjupdates+}

bmjupdates+ is a unique and free alerting service, designed to keep you up to date with the medical literature that is truly important to your practice.

bmjupdates+ will alert you to important new research and will provide you with the best new evidence concerning important advances in health care, tailored to your medical interests and time demands.

Where does the information come from?

bmjupdates+ applies an expert critical appraisal filter to over 100 top medical journals A panel of over 2000 physicians find the few 'must read' studies for each area of clinical interest

Sign up to receive your tailored email alerts, searching access and more..

www.bmjupdates.com 